\title{
Optical cell sorting with multiple imaging modalities
}

\author{
Banas, Andrew; Carrissemoux, Caro; Palima, Darwin; Glückstad, Jesper
}

\section{Published in:}

Proceedings of SPIE

Link to article, DOI:

$10.1117 / 12.2254358$

Publication date:

2017

Document Version

Publisher's PDF, also known as Version of record

Link back to DTU Orbit

Citation (APA):

Banas, A., Carrissemoux, C., Palima, D., \& Glückstad, J. (2017). Optical cell sorting with multiple imaging modalities. In Proceedings of SPIE: Complex Light and Optical Forces XI (Vol. 10120). [1012018] SPIE - The International Society for Optical Engineering. Proceedings of SPIE - The International Society for Optical Engineering https://doi.org/10.1117/12.2254358

\section{General rights}

Copyright and moral rights for the publications made accessible in the public portal are retained by the authors and/or other copyright owners and it is a condition of accessing publications that users recognise and abide by the legal requirements associated with these rights.

- Users may download and print one copy of any publication from the public portal for the purpose of private study or research.

- You may not further distribute the material or use it for any profit-making activity or commercial gain

- You may freely distribute the URL identifying the publication in the public portal 


\title{
Optical cell sorting with multiple imaging modalities
}

\author{
Andrew Bañas ${ }^{1,2}$, Caro Carrissemoux ${ }^{3}$, Mark Villangca ${ }^{1}$, Darwin Palima ${ }^{1}$, and Jesper Glückstad ${ }^{1,2}$ \\ ${ }^{1}$ Department of Photonics Engineering, Technical University of Denmark, Oersted Plads 343, DK-2800 Kgs. Lyngby, \\ Denmark \\ ${ }^{2}$ OptoRobotix ApS, Scion DTU, Diplomvej 381, DK-2800 Kgs. Lyngby, Denmark \\ ${ }^{3}$ Ghent University, St. Pietersnieuwstraat 33, 9000 Gent, Belgium
}

\begin{abstract}
Early detection of diseases can save lives. Hence, there is emphasis in sorting rare disease-indicating cells within small dilute quantities such as in the confines of lab-on-a-chip devices. However, before diseased cells can be studied in isolation, it is necessary to identify them against normal healthy cells. With the richness of visual information, a lot of microscopy techniques have been developed and have been crucial in biological studies. To utilize their complementary advantages we adopt both fluorescence and brightfield imaging in our optical cell sorter. Brightfield imaging has the advantage of being non-invasive, thus maintaining cell viability. Fluorescence imaging, on the other hand, takes advantages of the chemical specificity of fluorescence markers and can validate machine vision results from brightfield images. Visually identified cells are sorted using optical manipulation techniques. Scattering forces from beams actuated via efficient phase-only efficient modulation has been adopted. This has lowered the required power for sorting cells to a tenth of our previous approach, and also makes the cell sorter safer for use in clinical settings. With the versatility of dynamically programmable phase spatial light modulators, a plurality of light shaping techniques, including hybrid approaches, can be utilized in cell sorting.
\end{abstract}

Keywords: Cell sorting, fluorescence microscopy, microscopy, phase-only light modulation, optical trapping and manipulation, machine vision, microfluidics

\section{INTRODUCTION}

It is often said that much of what we know about the world is acquired through the sense of sight. As such, developments in image acquisition covers a wide range of applications: from remote astronomical observations, consumer electronics via cameras that are now even more ubiquitous and down to microscopic imaging which lends itself as a very important and powerful tool for biological research. While constantly improving electronics and algorithms take care of what happens of the electrical signals that come from camera sensors, what happens between the observed sample and the camera sensor primarily lies in the domain of optics. Furthermore, imaging sensors that serve as the physical interface between optics and electronics still have plenty of room for improvement in terms of resolution, dynamic range and detection efficiency. Current physical constraints of detectors relays to what data can be acquired and subsequently processed. In such case, the quality of data that comes out of the computing or machine vision system largely depends on the quality of data that is fed into it.

Image data acquired for scientific measurements are different from common photographs as the experimentalist has freedom in altering what is being observed without being limited to what is aesthetically pleasing. Such physical manipulations are done in order to enhance features such as contrast and signal-to-noise ratio. Hence a wide variety of microscopy techniques are being practiced in research usage. If the experimentalist knows what too look for, the type of microscopy technique to be employed can already be pre-determined. Fluorescence microscopy is thus often employed in situations where the biologists already have an idea of what chemicals to look for and what fluorescence markers attach to these chemicals.

It is not necessarily the case, however, that the user already knows what to look for. In more open and explorative research, one wishes to make a statistical correlation between an observable effect being studied and a new type of "indicator" to be discovered. Such indicator might be a morphological feature, a chemical signature, a difference in coloration, contrast or something else. These indicators need not be something immediately seen through visual inspection of isolated measurements but instead might be something that is eventually uncovered by a learning machine vision system analyzing large amounts of image data. As different microscopy imaging techniques acquire different types of image data, choosing techniques that complement each other ensures that features that are not observable with 
just one of these techniques are not missed out. Furthermore, cross-validations and possible correlations between these parallel imaging techniques can reinforce the reliability of such measurements.

It is important to detect diseases as early as possible, in other words, while a would-be patient is still apparently healthy. Early detection ensures that such disease can be dealt with immediately before they reach a more advanced stage wherein the patient may already be suffering. Unfortunately, early detection also poses a challenge as there would be much less indicators to be detected. Without obvious manifestation of symptoms, it becomes necessary to perform observations at the level of individual cells and identify a handful of disease-indicating cells amongst many millions of other healthy ones. Such early detection can be applicable to rare circulating tumor cells, or rare anemia in the blood and therefore can be potentially useful in taking preventive measures before the condition worsens ${ }^{3}$.

Given the importance of cell sorting in the early detection of diseases, devices for studying cells are being developed as early as the $60 \mathrm{~s}^{4}$. For example, one established method for cell sorting is based on flow cytometry or fluorescenceactivated cell sorting systems. Conventional flow cytometry works by queuing cells in a single line so they can be individually analyzed. An alternative based on optical fractionation ${ }^{5}$ was also proposed for sorting. Sorting is possible as particles with different properties have characteristic deflections as they flow through an optical lattice. As particle motion in fractionation is not actively controlled, careful calibration of the optical lattice is required. This might also be less suitable if the desired cells have identifying features that are still unknown or do not affect the flow characteristics of the cell.

We have taken a different approach on observing a stream of cells within a wider field of view $\left(\sim 100 \mathrm{x} 100\right.$ microns $\left.^{2}\right)$ then sorting them by using optical scattering forces as the driving mechanism ${ }^{6}$. Machine vision is used to automate the detection of specific cells. Once detected, cells are directly pushed into another stream for later collection and study. The cell sorting approach is illustrated in Figure 1. This approach may be compared with quality control of products as they are picked out from an assembly line conveyor, in which case, machine vision is also employed. Machine vision had been successfully used to identify objects and automate tasks as early as the 80 's wherein it is applied in sorting in quality control, automated manufacturing, unmanned vehicles among many others. Until today, with the constant improvements and increasing use of processing hardware and image recognition algorithms, and with the increasing amount of calibration image data, machine vision is actively used in diverse fields such as gaming and computing, unmanned vehicles, and person identification. Applied to optical manipulation, we use machine vision to locate cells that deviate from normal healthy ones and then activate optical traps based on the cell's position enabling automated manipulation $^{7}$ or dynamic stabilization ${ }^{8}$.

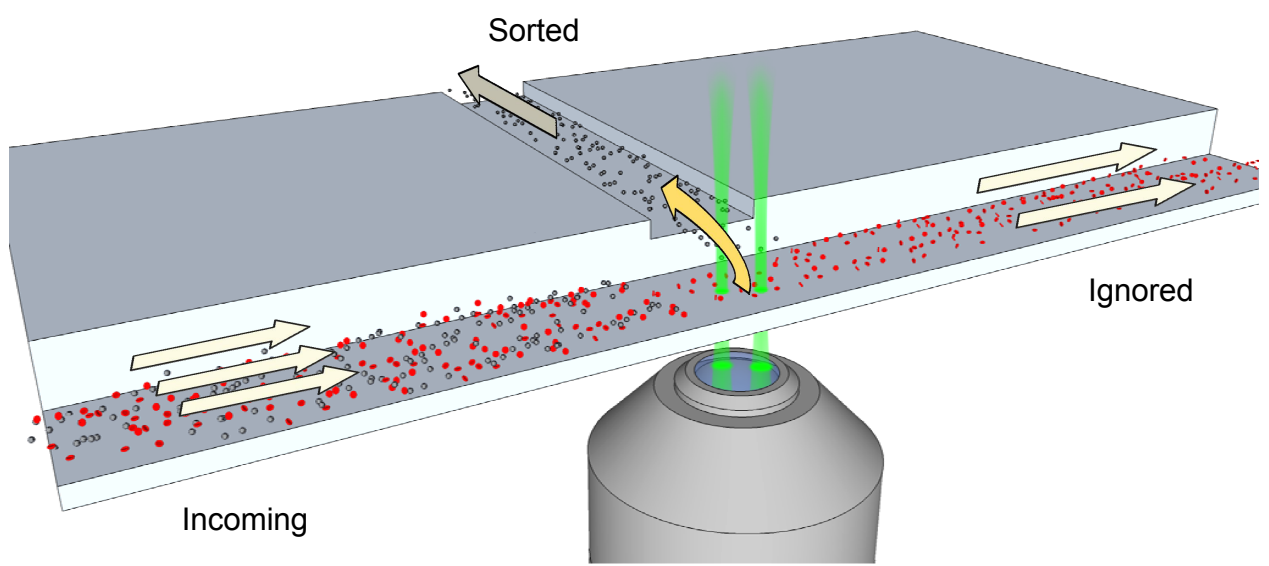

Figure 1. Sorting principle of the cell-BOCS. A sample of mixed cells flows through a detection window that is viewed through the microscope. Cells that fit a certain criterion are located via image processing. Optical traps catapult the identified cells to push them to another flow channel where they are sorted out.

Our cell sorting platform, the Bio-Optofluidic Cell Sorter ${ }^{9}$ (cell-BOCS), is therefore designed to utilize machine vision to search for abnormalities in cells. Visible features on cell samples such as morphology, size or color are used as a basis of discrimination. In addition to standard brightfield imaging, a fluorescence imaging channel can also run in parallel. As fluorescence is available, chemical features that do not necessarily correlate with morphological features can also serve 
as a basis for detection. The cell-BOCS's gentle sorting is based on low numerical aperture catapulting optical traps ${ }^{10}$. This gentle approach also preserves the cells viability for later studies and allowing the possibility of culturing the selected cells to increase their numbers and observe their reactions to possible treatments.

\section{EXPERIMENTS}

The cell-BOCS setup is shown in Figure 2. Two cameras (Basler) can simultaneously view the sample through the light that is split through the dichroic mirror. A more sensitive camera is used to view the fluorescence image from the light passing through the dichroic. A higher resolution camera is used to view the brightfield image formed by the light that is deflected off the dichroic filter. Besides providing complementary visual information of the samples, the brightfield image allows practical operations such as laser alignment and sample positioning to be performed even in the absence of visual cues from the fluorescence image.

For this experiment, the laser beam shaping of the optical traps is based on simple blazed gratings encoded on a phaseonly spatial light modulator. This is a more efficient modification of our previous test demonstrations where we used binary gratings encoded on a consumer projector ${ }^{11}$. Compared to the binary grating approach using amplitude modulation, the current approach is about 10x more efficient, lowering the power of the laser. This also leads to a safer operation of the device. Other efficient light shaping methods such as $\mathrm{GPC}^{12,13}$ or matched filtering $\mathrm{GPC}^{14,15}$ can be incorporated later due to the modular approach of the cell-BOCS's the laser beam shaping engine. Laser light $(\lambda=1070 \mathrm{~nm})$ that is shaped by the SLM is subsequently focused into the microfluidic chip at locations determined using computer vision.

To test proof of principle fluorescence imaging, yeast cells are used due to its simpler staining protocol. Targeted cells are optically sorted in a plastic microfluidic chip (Epigem) having a crossing similar to Fig. 1. Channels containing the cell samples and the destination buffer intersect at this analysis window. The cameras are used to monitor all the cells passing through the window. Image processing is performed to the grabbed frames to subsequently detect cells that fit certain morphological parameters set by the user. The SLM is updated in real time based on the location of the detected cells. The dynamically programmed light distribution then pushes the targeted cells away from the input stream, pushing them into the collection stream. After isolation, the sorted cells can be collected later for further analysis.

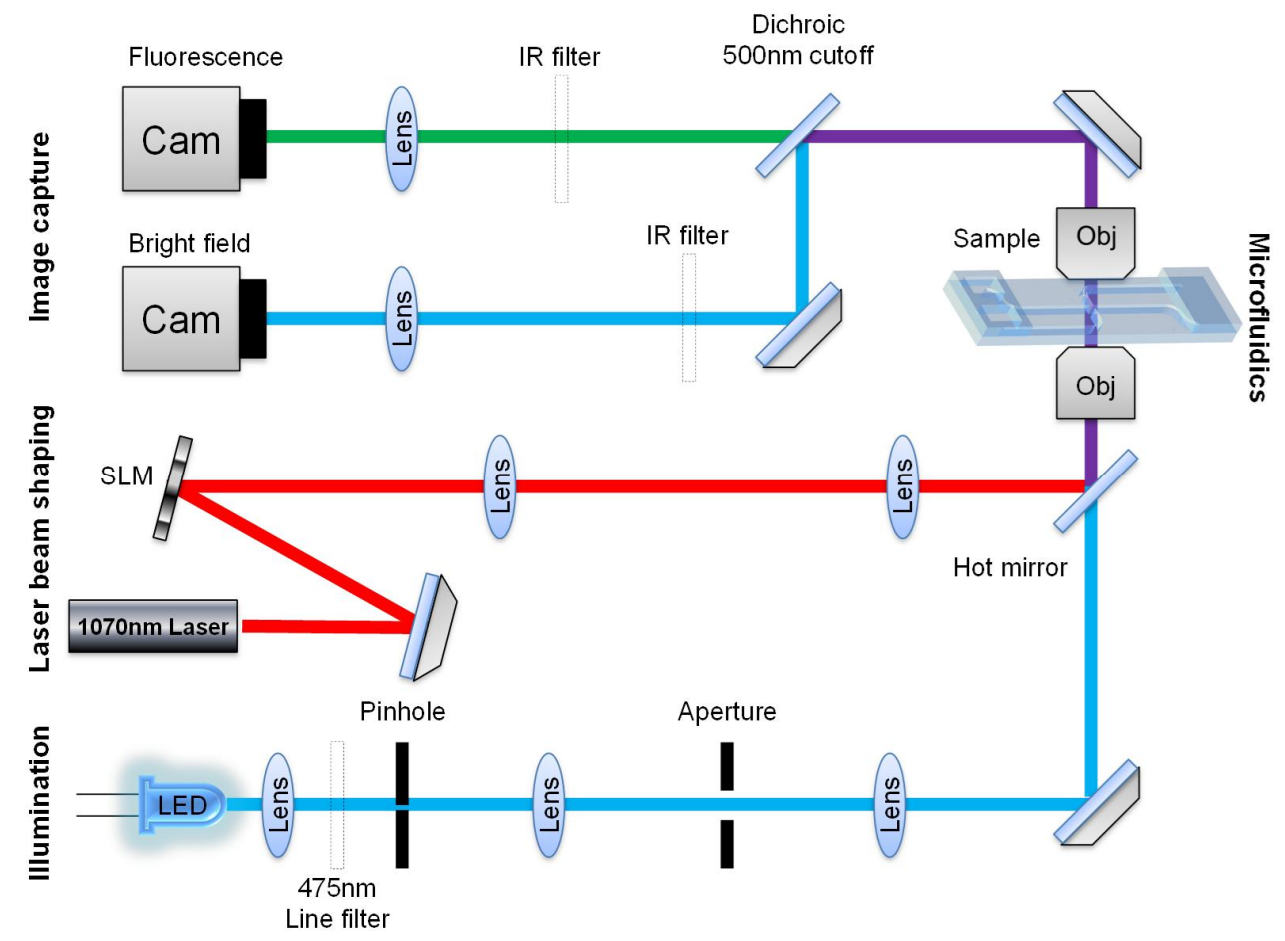

Figure 2. Schematic of the optical part of the cell-BOCS. 


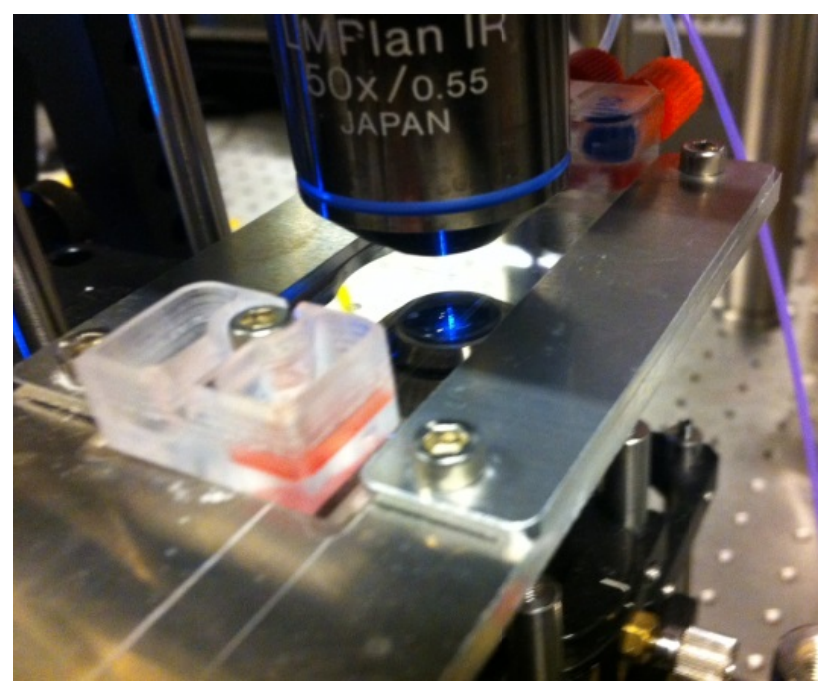

Figure 3. Microfluidic chip loaded with test cell samples and aligned in the optical path for imaging and laser based catapulting in the cell-BOCS.

\subsection{Results}

A close-up image of the microfluidic chip is shown in Figure 3. To minimize the introduction of air bubbles, the sample and buffer reservoirs are directly located on the chip instead of being connected to remote containers via extra tubing as in our previous work ${ }^{11}$. Syringe pumps pull the fluid out of the chip in order to establish a controlled flow where a stream of cells can be observed through a top microscope objective lens. The shaped laser beams that push the cell to the upper stream come from the lower objective lens. These trapping lasers are filtered from the camera view.

Side-by-side results of the simultaneous brightfield and fluorescence image captures are shown in Figure 4. Bright field imaging is obtained by illuminating the sample with a collimated LED source. Using switchable filters, the same objective is used for fluorescence imaging. Immunofluorescent labeled yeast cells are illuminated with $475 \mathrm{~nm}$ light. Emission above $500 \mathrm{~nm}$ passes through a dichroic filter and then is gathered by the camera. In practical cases it is anticipated that only a few cells would have a fluorescence emission and the fluorescence image would be dark and empty most of the time. Hence, the brightfield image gives a clue of what is going on within the sample environment even in the absence of fluorescent samples.

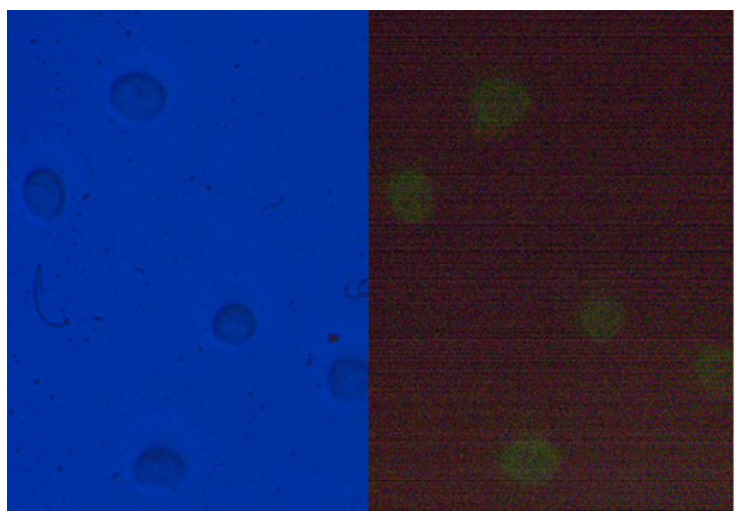

Figure 4. Simultaneously captured bright field and fluorescence imaging of immunoflurescent labeled yeast cells.

Another advantage of having an alternate imaging modality is the availability of higher contrast image captures for image processing. Despite the presence of noise at high camera gains due to weak fluorescence signals, there is enough contrast for the image processing routines to identify the cells as shown in Figure 5. In this case, the camera images are 
post processed via binarization, then the regions of white pixels are identified as individual cells as indicated by the red overlays on top of the fluorescence image.
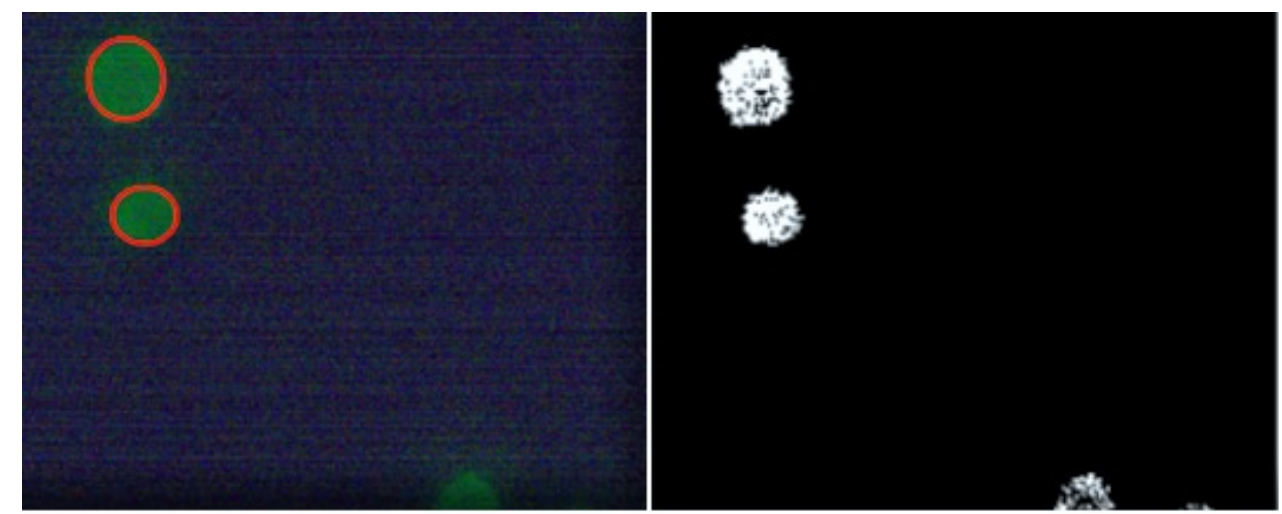

Figure 5. Detection and image processing of yeast cells stained with AlexaFluor 488 dye. The left side shows the camera capture with red overlays identifying the cells. The binarized post-processed image is shown in the right.

\section{CONCLUSIONS}

We have shown how the use of different imaging modalities assists in the operation of an optical cell sorter. For optimal performance, advances in image processing and acquisition should be met by advances optics based image enhancement. Different microscopy imaging modalities are also necessary to adapt to the variety of samples to be studied. We have used brightfield imaging which has the advantage of being non-invasive, and of visualizing the cell morphology. Complementary to this, fluorescence imaging takes advantages of the chemical specificity of fluorescence markers and can validate machine vision results from brightfield images.

\section{ACKNOWLEDGEMENTS}

This work has been supported by the Combined Molecular Microscopy for Therapy and Personalized Medication in Rare Anaemias Treatments (CoMMiTMent) FP7 collaborative project (Grant agreement number: 602121). We also acknowledge Hamamatsu Photonics K.K. Central Research Laboratory for assistance with the spatial light modulator, Epigem Ltd for lending their expertise in microfluidic device fabrication and Arivis AG for their contributions in the software used in the experiments.

\section{REFERENCES}

[1] Fu, A. Y., Spence, C., Scherer, A., Arnold, F. H.., Quake, S. R., “A microfabricated fluorescence-activated cell sorter," Nat Biotech 17(11), 1109-1111, Nature America Inc. (1999).

[2] Glückstad, J., "Sorting particles with light,” Nat. Mater. 3, 9-10 (2004).

[3] Sleijfer, S., Gratama, J.-W., Sieuwerts, A. M., Kraan, J., Martens, J. W. M.., Foekens, J. A., "Circulating tumour cell detection on its way to routine diagnostic implementation?," Eur. J. Cancer 43(18), 2645-2650, Elsevier (2007).

[4] Fulwyler, M. J., "Electronic separation of biological cells by volume," Science (80-. ). 150(3698), 910-911, American Association for the Advancement of Science (1965).

[5] MacDonald, M. P., Spalding, G. C.., Dholakia, K., "Microfluidic sorting in an optical lattice," Nature 426(6965), 421-424 (2003).

[6] Ashkin, A., “Acceleration and Trapping of Particles by Radiation Pressure,” Phys. Rev. Lett. 24(4), 156-159, American Physical Society (1970).

[7] Tanaka, Y., Kawada, H., Hirano, K., Ishikawa, M.., Kitajima, H., "Automated manipulation of non-spherical micro-objects using optical tweezers combined with image processing techniques," Opt. Express 16(19), 1511515122, Optical Society of America (2008). 
[8] Tauro, S., Bañas, A., Palima, D.., Glückstad, J., "Dynamic axial stabilization of counter-propagating beam-traps with feedback control.," Opt. Express 18(17), 18217-18222 (2010).

[9] Bañas, A., Palima, D., Pedersen, F.., Glückstad, J., "Development of a compact Bio-Optofluidic Cell Sorter," Proc. SPIE Vol. 8274 8274, 82740N (2012).

[10] Ulriksen, H.-U., Thogersen, J., Keiding, S., Perch-Nielsen, I. R., Dam, J. S., Palima, D. Z., Stapelfeldt, H.., Gluckstad, J., "Independent trapping, manipulation and characterization by an all-optical biophotonics workstation," J. Eur. Opt. Soc. Rapid Publ. 3 (2008).

[11] Bañas, A., Palima D., Villangca, M., Glückstad, J., "Cell sorting using efficient light shaping approaches," Proc. SPIE 9764, Complex Light and Optical Forces X, 97640F (2016).

[12] Glückstad, J.., Palima, D. Z., Generalized Phase Contrast: Applications in Optics and Photonics, Springer Series in Optical Sciences (2009).

[13] Rodrigo, P. J., Gammelgaard, L., Bøggild, P., Perch-Nielsen, I.., Glückstad, J., “Actuation of microfabricated tools using multiple GPC-based counterpropagating-beam traps.,” Opt. Express 13(18), 6899-6904 (2005).

[14] Bañas, A., Palima, D.., Glückstad, J., "Matched-filtering generalized phase contrast using LCoS pico-projectors for beam-forming.," Opt. Express 20(9), 9705-9712 (2012).

[15] Perch-Nielsen, I., Palima, D., Dam, J. S.., Glückstad, J., "Parallel particle identification and separation for active optical sorting," J. Opt. A Pure Appl. Opt. 11(3), 034013 (2009). 